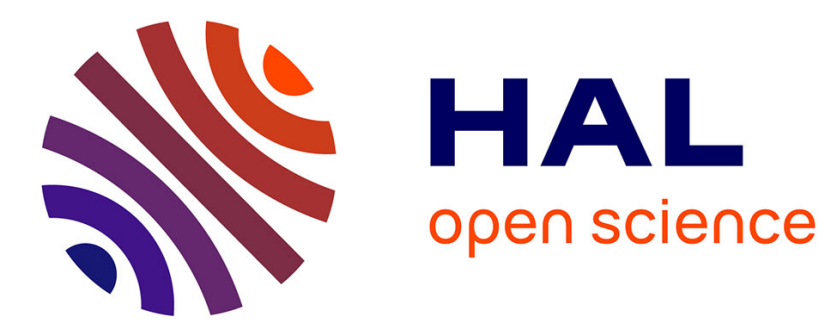

\title{
Mirrorless optical parametric oscillators with stitching faults: Backward downconversion efficiency and coherence gain versus stochastic pump bandwidth
} Carlos Montes, Bernard Gay-Para, Marc De-Micheli, Pierre Aschieri

\section{- To cite this version:}

Carlos Montes, Bernard Gay-Para, Marc De-Micheli, Pierre Aschieri. Mirrorless optical parametric oscillators with stitching faults: Backward downconversion efficiency and coherence gain versus stochastic pump bandwidth. 2014. hal-01074554

\section{HAL Id: hal-01074554 \\ https://hal.science/hal-01074554}

Preprint submitted on 14 Oct 2014

HAL is a multi-disciplinary open access archive for the deposit and dissemination of scientific research documents, whether they are published or not. The documents may come from teaching and research institutions in France or abroad, or from public or private research centers.
L'archive ouverte pluridisciplinaire HAL, est destinée au dépôt et à la diffusion de documents scientifiques de niveau recherche, publiés ou non, émanant des établissements d'enseignement et de recherche français ou étrangers, des laboratoires publics ou privés. 


\title{
Mirrorless optical parametric oscillators with stitching faults: Backward downconversion efficiency and coherence gain versus stochastic pump bandwidth
}

\author{
Carlos Montes*, Bernard Gay-Para, Marc de-Micheli, and Pierre Aschieri \\ Université Nice Sophia Antipolis, CNRS, Laboratoire de Physique de la Matière Condensée \\ (UMR 7336), F-06100 Nice, France \\ *Corresponding author: carlos.montes@unice.fr
}


Parametric downconversion in submicronic periodically poled (PP) quadratic materials allows the generation of signal and idler waves in opposite directions. The distributed feedback mechanism enables a backward mirrorless optical parametric oscillator (BMOPO), which has been experimentally realized using a periodically-poled KTP crystal with $800 \mathrm{~nm}$ periodicity. A remarkable spectral property of the BMOPO is that the bandwidth of the forward co-propagating wave is comparable to the pump bandwidth, whereas that of the backward wave is typically several orders of magnitude narrower. In the backward idler configuration realized in PPKTP the backward idler exhibits a coherence gain of two orders of magnitude. In this paper we will consider the backward signal configuration, where the effect of coherence enhancement of the backward signal wave is especially pronounced for exact group velocity matching of the co-propagating pump and idler waves. This QPM scheme requires a $335 \mathrm{~nm}$ scale poling periodicity which may be achieved in GaN waveguides, combining e-beam lithography and epitaxy. However, during the lithography step necessary to obtain the PP material, stitching faults may occur. The BMOPO device is then formed by a sequence of 200 microns long PP elements stuck together and we assume that the stitching faults do not exceed half of the poling period, in order to prevent the parametric reverse process. The original three-wave QPM model with the explicit periodicity of the nonlinear parametric coupling is necessary for taking into account the periodicity faults in the junctions. Thanks to the High Performance Computing facility available at Nice University, we analyse the parametric downconversion efficiency and the coherence gain of the backward signal versus the stochastic pump bandwidth in a large pump bandwidth range. We show that if the stitching faults do not exceed a quarter of the poling period, i.e. less than $80 \mathrm{~nm}$, which is technically feasible, the efficiency is comparable to the BMOPO in the perfect PP material. In any case, for an incoherent pump of broad spectral bandwidth we obtain a coherence gain of almost three orders of magnitude. (c) 2014 Optical Society of America

OCIS codes: 030.1640, 190.4410, 190.4970, 190.5970, 230.4320

\section{Introduction}

Optical parametric oscillators based on periodically poled (PP) nonlinear quadratic materials, with submicronic poling period allow the generation of quasi-phase-matched (QPM) signal and idler waves in opposite directions $[1,2]$. The distributed feedback mechanism enables backward mirrorless optical parametric oscillation (BMOPO). This has been experimentally 
realized in the backward idler configuration using a periodically-poled $\mathrm{KTiOPO}_{4}(\mathrm{PPKTP})$ crystal with $\Lambda_{G}=800 \mathrm{~nm}$ periodicity as a QPM nonlinear medium [3]. A remarkable spectral property of the BMOPO is that the co-propagating forward wave has a bandwidth comparable to the pump bandwidth, whereas that of the backward wave is typically several orders of magnitude narrower. Two orders of magnitude have been obtained in this backward idler configuration, recently demonstrated both experimentally and numerically for a pump wave whose bandwidth is stretched by deterministic phase modulation in a PPKTP BMOPO for relatively narrowband (1.2 THz) linearly-chirped pump pulses [4] and relatively broadband (4 THz) pump pulses too [5].

In this paper we will consider the backward signal configuration, where the effect of coherence enhancement of the backward signal wave is especially pronounced owing to the possibility of exact group velocity matching of the forward co-propagating pump and idler waves. But the short nanometric periods required for QPM have been only experimentally achieved, up to now in GaN, combining e-beam lithography and epitaxy [6]- [8]. Asymmetric GaN waveguides grown on (0001) sapphire by molecular beam epitaxy or by metal-organic chemical vapor deposition are promising structures for applications in nonlinear optics. Periodically poled GaN (PPGaN) was used for second harmonic generation [6] and the possibility to grow submicronic PP GaN films [7] opens the door for parametric counterpropagating oscillation thanks to reasonably large second order susceptibility $\left(\chi_{33}^{(2)}=10.6 \mathrm{pm} / \mathrm{V}\right.$ measured in [8]), comparable to that of KTP.

However, during the fabrication process of the PPGaN waveguide, stitching errors cannot be avoided for centimeter long interaction lengths. Indeed, during the lithography step necessary to obtain the PP material, the realization of centimeter long devices presenting a submicronic periodicity is not possible without moving the sample under the writing beam. This displacement introduces the stitching faults. We have studied the dynamics in a PPGaN BMOPO formed by a sequence of 200 microns long PP elements (PPGaN bricks) stuck together, so that the stitching faults do not exceed half of the poling period $\Lambda_{G}$ in order to avoid the switch from parametric downconversion to the reverse process of sum frequency generation, which combines signal and idler to create pump $[9,10]$. The defects will also affect the parametric downconversion efficiency and the phenomenon of coherence enhancement which decrease as the amplitude of the perturbations increases. In order to take into account the periodicity perturbations leading to phase shifts in the junctions between the perfect QPM PP segments, we must consider the original QPM model [11,12] with the complete explicit dependece of the nonlinear coupling parameter, without substituting the grating periodicity by an effective quadratic nonlinearity, i.e. without introducing the usual exact phase-matching approximation [13].

Our aim here is to study the coherence transfer for a broad bandwidth stochastic pump. 
The generation of a coherent wave in the process of parametric down-conversion of an incoherent wave by the convection-induced phase-locking mechanism has been the object of theoretical and numerical studies [14]- [18]. In accordance with this mechanism, the phase modulation in the pump is coherently transferred to the forward parametric wave, while the backward wave retains a narrow bandwidth and high coherence. Some recent studies show the parametric amplification of the signal wave by conversion of a broadband incoherent pump to the group velocity matched idler wave in the forward three-wave interaction [19]- [21] and using the generalized nonlinear envelope equation [22].

Thanks to the 1200 multiprocessor computer facility (HPC) of the "Centre de Calcul Interactif" hosted by the "Université Nice Sophia Antipolis", the coherence transfer mechanism is studied here for broad incoherent pump pulses in a large spectral bandwidth range $[0.08 \mathrm{GHz}$ - 6.0 THz]. We analyse the downconversion efficiency versus the stochastic pump bandwidth for fragmented PPGaN BMOPOs whose stitched junctions have a perturbation length lower than $\Lambda_{G} / 2$, and we compare them to the perfect PPGaN BMOPO. For a stochastic pump of broad spectral bandwidth and maximum perturbation length of $80 \mathrm{~nm}$, which is technically feasible, we obtain a coherence gain of almost three orders of magnitude.

\section{BMOPO model for a QPM fragmented waveguide}

In a BMOPO, the pump wave is downconverted into forward-propagating and backwardpropagating parametric waves, in accordance with energy conservation, $\omega_{p}=\omega_{s}+\omega_{i}$ and counterpropagating quasi-phase matching, $k_{p}+k_{s}-k_{i}=K_{G}$, where $j=p, s, i$ denote the pump, the backward signal and the forward idler waves, respectively. $K_{G}=2 \pi / \Lambda_{G}$ is the grating wave vector given by $\Lambda_{G}$, the modulation period of the second-order nonlinear coefficient. The coherence enhancement effect of the backward wave is especially pronounced when the pump wavelength and the index profile of the waveguide are chosen so that the group velocities of the pump and the forward idler wave are exactly matched [15]. This configuration may be achieved in a GaN waveguide with a group velocity dispersion modified by a particular profile of the refractive index [17], a technique widely used in fibers to compensate the material dispersion with the waveguide dispersion [23]. For $z$-polarized waves in PPGaN, matching group velocities can be achieved by designing the index profile so that the pump and the forward wave are on different sides of the maximum on the group-velocity curve shown in Fig. 1. This shape of group-velocity curve may be obtained in the guided PPGaN structure composed of a ridge GaN waveguide of $2 \mu \mathrm{m}$ in the width and $1.8 \mu \mathrm{m}$ in the depth on a AlInN buffer which has been realized on sapphire substrate by epitaxy. The Sellmeier relation given in [8] is used for material dispersion, and finite waveguide dispersion corrections [17] yield the group-velocity curve of Fig. 1 This may be realized in the GaN structure combining e-beam lithography and epitaxy $[7,8]$. To fulfil QPM condition with the 
set of wavelengths chosen here we need a periodicity of the grating of $\Lambda_{G}=335 \mathrm{~nm}$.

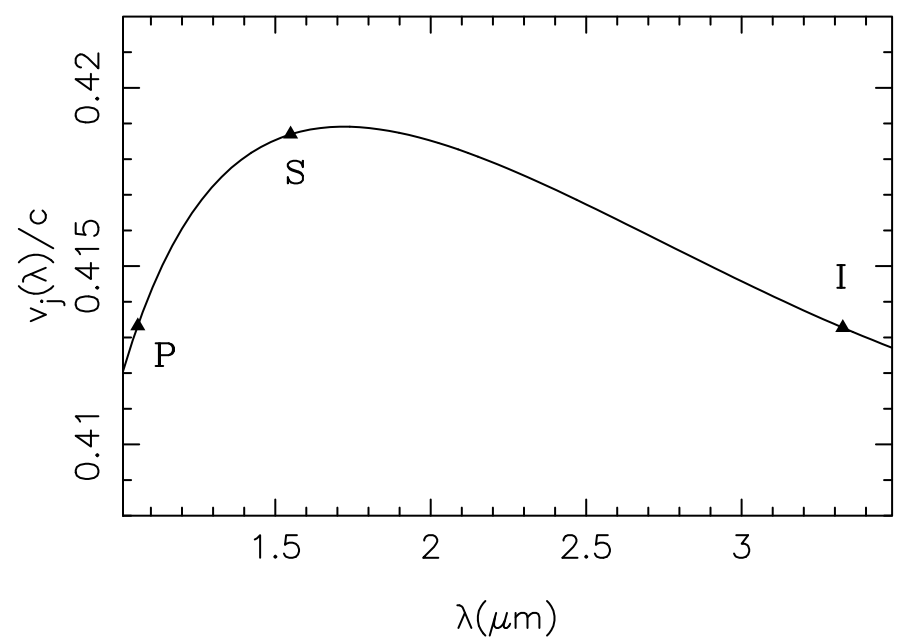

Fig. 1. Group velocity for z-polarized waves in GaN as function of the wavelength, calculated from the Sellmeier expansion [8] with a particular profile of the refractive index of the guide [17]. The triangles mark the configuration of perfectely matched pump and idler group velocities $\left(\left|\mathrm{v}_{\mathrm{p}}-\mathrm{v}_{\mathrm{i}}\right|=0\right)$, and are labeled $\mathbf{P}$ for the pump, $\mathbf{S}$ for the backward signal, and $\mathbf{I}$ for the idler.

The pump intensity required to reach threshold for BMOPO operation, $I_{p, t h}$, can be estimated from the plane-wave monochromatic model for the undepleted pump parametric approximation [2] as $I_{p, t h}=c \varepsilon_{0} n_{p} n_{f} n_{b} \lambda_{f} \lambda_{b} / 2 L^{2} d_{e f f}^{2}$, where $c$ is the speed of light in vacuum, $\varepsilon_{0}$ is the permittivity of free space, $n_{j}$ denotes the refractive indices, $\lambda_{j}$ denotes the backward and forward wavelengths, $L$ is the nonlinear interaction length and $d_{\text {eff }}$ is the effective quadratic nonlinearity.

Numerical simulations of the nonlinear three-wave interactions in a BMOPO solve the coupled three wave equations in the slowly-varying-envelope approximation. The parameters used in the model are those of a periodically poled GaN waveguide (PPGaN) grown by epitaxy on sapphire substrates [6] and presenting submicronic periodicity of the $\chi^{(2)}$ coefficient [7]. The effective nonlinear coefficient is then given by $d_{e f f}=(2 / \pi) \chi_{33}^{(2)}$. Depending on the experimental measurement of $\chi_{33}^{(2)}, d_{\text {eff }}$ varies from $7 \mathrm{pm} / \mathrm{V}$ [8] to $3.8 \mathrm{pm} / \mathrm{V}[24]$ $\left( \pm 45 \%\right.$ of accuracy). For the mechanism studied here we will take the value of $d_{\text {eff }}=5$ $\mathrm{pm} / \mathrm{V}$. In order to account for stitching faults in the joints, or periodicity perturbations, simulated through phase shifts between the perfect QPM segments, we call on the original QPM model [11,12], without substituting the grating periodicity of the nonlinear coupling parameter by an effective quadratic nonlinearity. Indeed, we have shown - within the exact 


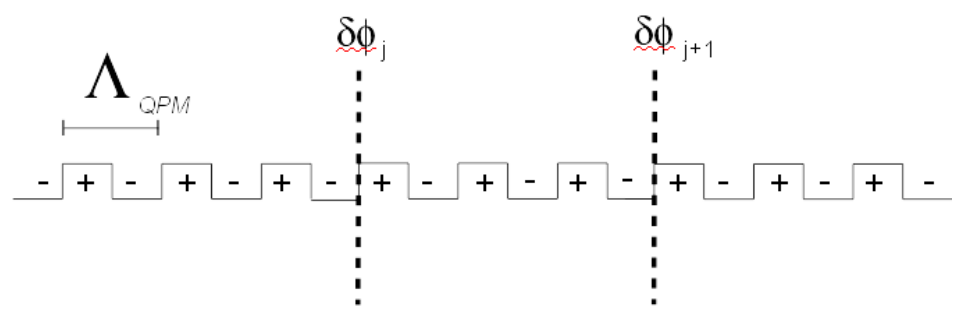

Fig. 2. The quasi-phase-matching (QPM) is obtained by a phase-reversal sequence of the ferroelectric domains with a grating periodicity $\Lambda_{G}=\Lambda_{Q P M}=$ $335 \mathrm{~nm}$. The segmented backward MOPO is composed of a train of periodically poled GaN elements of length $\ell=195 \mu \mathrm{m}$. Stitching faults between the N PP elements stuck together, are simulated by a phase-shift perturbation $\delta \phi_{m}(x)(m=1, \mathrm{~N}-1)$ at each joint $m$, whose amplitude is randomly distributed in the interval $(0, p \pi)$, with $p<1$ to avoid the parametric reverse process. It is associated to a stitching length $\Lambda_{G} \delta \phi_{m}(x) /(2 \pi)$.

phase-matching model -, that the parametric downconversion efficiency and the coherence enhancement is as high as for the perfect single piece PP waveguide whatever be the number of segments separated by uniformly polarized sections [13]. This would correspond to an ideal case where the separation of the perfect PP segments has the length of an entire number of grating periods.

The field amplitudes, $\mathbf{A}_{\mathbf{j}}(\mathbf{j}=\mathbf{p}, \mathbf{s}, \mathbf{i})$, of the pump and the parametric backward signal wave and forward idler wave, evolve with the coupled equations:

$$
\begin{aligned}
\left(\partial_{t}+\mathrm{v}_{\mathrm{p}} \partial_{x}+\gamma_{p}+i \beta_{p} \partial_{t t}\right) \mathbf{A}_{\mathbf{p}} & =-\sigma_{\mathrm{p}} \cos \left[K_{G} x+\delta \phi_{m}(x)\right] \mathbf{A}_{\mathbf{s}} \mathbf{A}_{\mathbf{i}} \exp (i \Delta K x) \\
\left(\partial_{t}-\mathrm{v}_{\mathrm{s}} \partial_{x}+\gamma_{s}+i \beta_{s} \partial_{t t}\right) \mathbf{A}_{\mathbf{s}} & =\sigma_{\mathrm{s}} \cos \left[K_{G} x+\delta \phi_{m}(x)\right] \mathbf{A}_{\mathbf{p}} \mathbf{A}_{\mathbf{i}}^{*} \exp (-i \Delta K x) \\
\left(\partial_{t}+\mathrm{v}_{\mathrm{i}} \partial_{x}+\gamma_{i}+i \beta_{i} \partial_{t t}\right) \mathbf{A}_{\mathbf{i}} & =\sigma_{\mathrm{i}} \cos \left[K_{G} x+\delta \phi_{m}(x)\right] \mathbf{A}_{\mathbf{p}} \mathbf{A}_{\mathbf{s}}^{*} \exp (-i \Delta K x),
\end{aligned}
$$

where $\gamma_{j}$ and $\beta_{j} \equiv \mathrm{v}_{\mathrm{j}} \beta_{2, j} / 2$ are the damping and dispersion coefficients, and $\sigma_{j}=$ $2 \pi d_{e f f} \mathrm{v}_{\mathrm{j}} / \lambda_{j} n_{j}$ are the nonlinear coupling coefficients in the PP elements, with the spatial period $\Lambda_{G}=2 \pi / K_{G}$ of the periodic poled $\chi_{33}^{(2)}$ domains and $\Delta K=k_{p}+k_{s}-k_{i}$. By expanding the second-order susceptibility in Fourier series and retaining the first order we obtain $\chi_{F}^{(2)} \simeq \chi_{e f f}^{(2)} \cos \left(K_{G} x\right)[2]$. Inside the PP elements $\sigma_{\mathrm{j}} \cos \left(K_{G} x\right)$ is periodically perfect, so that QPM is not affected by fluctuating nonlinearity [31] or random effects [25,26], which would lead to effective nonlinear losses. The stitching faults introduced through finite phase shift perturbations $\delta \phi_{m}(x)(m=1, \mathrm{~N}-1)$ between the N stuck PP segments (cf. Fig. 2) are only located at each junction $m$. We take them to be randomly distributed in the interval $(0, p \pi))$, with $p<1$ to avoid the parametric reverse process. In order to preserve efficient paramet- 
ric amplification and coherence enhancement we will limit ourselves to phase perturbations $\delta \phi_{m}(x) \leq \pi / 2$, which correspond to perturbation lengths $\leq \Lambda_{G} / 4$. By expanding the secondorder susceptibility of the domains in Fourier series and retaining the first order grating we obtain $\chi_{F}^{(2)} \simeq \chi_{e f f}^{(2)} \cos \left(K_{G} x\right)$ [2]. The exact phase-matched model equations [13] are obtained from equations (1) by imposing the matching condition $\exp \left(i K_{G} x\right)=\exp (i \Delta K x)$ but differ by non-phase-matched terms. As known [27], the parametric growth rate governed by (1) is lower than that for the exact QPM model.

The input parameters in the model are the properties of the nonlinear medium and the pump amplitude at the input face, $\mathbf{A}_{\mathbf{p}}(x=0, t)$, generating outputs of $\mathbf{A}_{\mathbf{p}}(x=L, t)$, $\mathbf{A}_{\mathbf{i}}(x=L, t)$ and $\mathbf{A}_{\mathbf{s}}(x=0, t)$. The nonlinear counterpropagation dynamics in the quadratic $\mathrm{BMOPO}$ in the presence of group-velocity dispersion, which introduces second-order time derivatives, makes use of the numerical scheme which combines the trajectories method and 4th-order Runge Kutta algorithm for the treatment of the nonlinear counterpropagating three-wave interaction, extensively used for the treatment of stimulated Brillouin backscattering problems $[28,29]$, with fast Fourier transformation (FFT) to account for the groupvelocity dispersion effects in the spectral domain [30].

The nonlinear medium is a GaN structured ensemble composed by a train of N periodicallypoled GaN elements of length $\ell$ disposed in a straight row as shown in Fig. 2. Simulations for this segmented structure of length $\mathrm{L}=\mathrm{N} \ell$, where a random phase-shift $\delta \phi_{m}(x)(m=1, \mathrm{~N}-$ 1) takes place at each jonction $\mathrm{m}$, representing the stitching fault between the $\mathrm{N}$ stuck PP segments, associated to a physical stitching length $\delta \mathrm{L}_{m}(x)=\Lambda_{G}\left(\delta \phi_{m}(x) /(2 \pi)\right.$, will be compared to the case of a one piece perfect PP medium of length $\mathrm{L}$ without phase-shifts. For $\mathrm{L}=64 \ell=1.25 \mathrm{~cm}$, each PP element has a length $\ell=195 \mu \mathrm{m}$. The waveguide has a section $\mathrm{S}_{\mathrm{eff}}=3.6 \mu \mathrm{m}^{2}$.

We choose the three QPM waves marked on the Fig. 1, and we model BMOPO operation with a broadband stochastic pump pulse with a FWHM temporal length of $150 \mathrm{ps}$, and where the spectral FWHM width $\Delta \nu_{p}(0)$ centered at $\lambda_{\mathrm{p}}=1.06 \mu \mathrm{m}$ varies in a large spectral bandwidth range $[0.08 \mathrm{GHz}-6.0 \mathrm{THz}]$, with $\mathrm{n}_{\mathrm{p}}=2.30856$, $\mathrm{v}_{\mathrm{p}} / \mathrm{c}=0.41337, \beta_{2, \mathrm{p}}=0.286 \mathrm{ps}^{2} / \mathrm{m}$; and with $\lambda_{\mathrm{s}}=1.56242 \mu \mathrm{m}, \mathrm{n}_{\mathrm{s}}=2.26580, \mathrm{v}_{\mathrm{s}} / \mathrm{c}=0.41872$, $\beta_{2, \mathrm{~s}}=0.0647 \mathrm{ps}^{2} / \mathrm{m} ; \quad \lambda_{\mathrm{i}}=3.29791 \mu \mathrm{m}, \quad \mathrm{n}_{\mathrm{i}}=2.12353, \quad \mathrm{v}_{\mathrm{i}} / \mathrm{c}=0.41337, \quad \beta_{2, \mathrm{i}}=-0.418 \mathrm{ps}^{2} / \mathrm{m}$. The QPM period as low as $\Lambda_{G}=335 \mathrm{~nm}$ may be realized in GaN by combining e-beam lithography and epitaxy [8].

The pump pulse is issued from a Gaussian stochastic process translationally invariant with zero mean $\left\langle\mathbf{A}_{\mathbf{p}}(\mathbf{x}=\mathbf{0}, \mathbf{t})\right\rangle$ and exponential autocorrelation function $\left\langle\mathbf{A}_{\mathbf{p}}\left(x=0, t^{\prime}+t\right) \mathbf{A}_{\mathbf{p}}^{*}(x=0, t)\right\rangle=\left|\mathbf{A}_{\mathbf{p}}^{\mathbf{0}}\right|^{2} \exp \left(-|t| / \tau_{c}\right)$, where $\tau_{c}=1 / \pi \Delta \nu_{p}$ is the correlation time. Note that, while the pump phase fluctuations are absorbed by the idler wave thanks to the phase-locking mechanism, the generation of the signal still remains affected by the 
intensity fluctuations of the pump. In the presence of small group velocity difference between the pump and the signal, which is the case for the forward OPO, pump intensity fluctuations are partly transferred to the signal component $[16,18]$. Conversely, in the presence of the strong group velocity difference of the backward MOPO, intensity fluctuations of the pump are averaged by the strong convection effect, which thus leads to an enhancement of the coherence of the backward wave [5] [13]. The higher the incoherence of the pump, the larger the amount of coherence transfer as studied in section V.B of Ref. [15].

Stochastic BMOPO operation in both continuous and segmented structures are simulated with a pump pulse of intensity $\mathrm{I}_{p}=4 \mathrm{GW} / \mathrm{cm}^{2}$, the BMOPO threshold intensity being $\mathrm{I}_{p, t h}$ $=2 \mathrm{GW} / \mathrm{cm}^{2}$. The peak power yields $140 \mathrm{~W}$, a pulsed energy of $20 \mathrm{~nJ}$, and a fluence of 0.6 $\mathrm{J} / \mathrm{cm}^{2}$ which remains below the damage threshold for GaN [32,33].

The resonant three-wave interaction inside the PPGaN BMOPO composed of the 64 PP segments is illustrated in Fig 3. We show in Fig 3(a), at time $t=254$ ns near the maximum of the nonlinear interaction, the forward stochastic pump amplitude, the induced stochastic forward idler amplitude and the resulting high coherent backward amplitude. The phaselocking behaviour inside the entire BMOPO length $\mathrm{L}$ is illustrated in Fig 3(b) and zoomed in the the first L/10 length in Fig 3(c). We remark that the forward idler phase is a replica (phase conjugate) of the stochastic pump phase, whereas the backward signal phase remains almost constant throughout the medium. As expected from the convection-induced phaselocking mechanism, even for the fragmented BMOPO, the stochastic phase modulation in the pump is essentially transferred to the forward idler, while the phase of the backward signal is almost constant.

To avoid other nonlinearities, such as stimulated Raman scattering, which is the most harmful nonlinearity competing with the parametric downconversion [3], we can further decrease the pump intensity and increase the interaction length. With $\mathrm{I}_{p}=2 \mathrm{GW} / \mathrm{cm}^{2}$ and a fluence of $0.3 \mathrm{~J} / \mathrm{cm}^{2}$, and for a pump depletion of $15 \%$ we attain a coherence gain $\Delta \nu_{p}(L) / \Delta \nu_{s}(0)=173$.

\section{Efficiency and coherence gain within the quasi-phase-matching model}

In order to account for stitching faults which are able to arise in the joints, simulated through phase shifts or finite perturbations between the perfect QPM segments we use the original QPM model of Eqs.(1), without substituting the grating periodicity of the nonlinear coupling parameter by an effective quadratic nonlinearity. Within this QPM model, the pump depletion or parametric downconversion efficiency versus initial pump bandwidth for the 64 times segmented BMOPO is compared to the one piece perfect PP waveguide shown in Fig 4(a), and repeated in the upper curves of Fig 5 and Fig 6 for direct comparison. In Fig 4(b), it 
is shown for random phase perturbations in the junctions $0<\delta \phi_{m}(x)<\pi / 4(m=1, \mathrm{~N}-1)$ and presents a relative efficiency of $93 \%$ with respect to the perfect PP waveguide shown in Fig 4(a). In the lower curve of Fig 5 it is shown for random phase perturbations in the junctions $0<\delta \phi_{m}(x)<\pi / 3(m=1, \mathrm{~N}-1)$ and the direct comparison to the upper curve for the perfect PP waveguide yields a relative efficiency of $87 \%$. In the lower curve of Fig 6 it is shown for random phase perturbations in the junctions $0<\delta \phi_{m}(x)<\pi / 2(m=1, \mathrm{~N}-1)$ and the direct comparison to the upper curve for the perfect PP waveguide yields a relative efficiency of $63 \%$. We obtain that the downconversion efficiency and the coherence enhancement are limited by the stitching defects as much as their length increase approaching $\Lambda_{G} / 2$. For $\Lambda_{G} / 2$ the downconversion efficiency vanishes. It is interesting to recall that for the ideal case where the stitching length is an entire number of $\Lambda_{G}$, the exact phase-matching model provides a parametric downconversion efficiency and a coherence signal enhancement as high as for the single piece perfect PP waveguide, provided that the sum of the PP segment lengths be equal to the length of the uniform PP waveguide [13]. Each point of the curves plotted in Fig 4(a)(b), Fig 5, and Fig 6, is a mean value over three different initial phases and requires 21 hours CPU numerical calculation due to the combined Runge-Kutta-FFT procedure [30]. Each curve contains 284 points and the total CPU time for one processor would be $21 \times 284=5964 \mathrm{CPU}$ hours for each curve. The use of High Performance Computing facility (a 1200 multiprocessor computer) has reduced this time by more than two orders of magnitude.

The apparent random variation of the pump depletion or downconversion efficiency around the mean linear fitting is due to stochastic resonances in the nonlinear medium associated to the initial phase. Its amplitude lowers when the mean value is performed over more initial phases.

The pump and the parametric spectra for the 64 times segmented BMOPO are illustrated in Fig 7 to Fig 9 for increasing pump bandwidth input and increasing phase-shift amplitude perturbation. As we can see, the higher the incoherence of the pump (larger spectral bandwidth), the larger the amount of coherence enhancement of the signal wave. By integrating the spectra, it is found that the conversion into parametric waves for the five chosen cases shown in Table 1, decrease with increasing pump bandwidth. For an incoherent pump spectrum of FWHM $\Delta \nu_{p}(\mathrm{~L})=4.54 \mathrm{THz}$, phase-shift amplitude perturbations in the joints $\leq \pi / 2$, and a pump depletion of 0.0256, Figure 9 shows a coherence gain $\Delta \nu_{p} / \Delta \nu_{s}=467$, which is comparable to the perfect PPGaN BMOPO. This maximum stitching of $\pi / 2$, i.e. $80 \mathrm{~nm}$ length, is technically attainable. 
Table 1. Parametric downconversion efficiency and coherence gain in the 64 times segmented GaN BMOPO with stitching faults of maximum $\pi / 4$, of maximum $\pi / 3$ (marked with ${ }^{*}$ ), and of maximum $\pi / 2$ (marked with ${ }^{* *}$ ).

\begin{tabular}{llllllll}
\hline $\begin{array}{l}\Delta \nu_{p}(0) \\
{[\mathrm{THz}]}\end{array}$ & $\begin{array}{l}\Delta \nu_{p}(L) \\
{[\mathrm{THz}]}\end{array}$ & $\begin{array}{l}\Delta \nu_{s}(0) \\
{[\mathrm{GHz}]}\end{array}$ & $\begin{array}{l}\Delta \nu_{i}(L) \\
{[\mathrm{THz}]}\end{array}$ & $1-\frac{I_{p}(L)}{I_{p}(0)}$ & $\frac{I_{s}(0)}{I_{p}(0)}$ & $\frac{I_{i}(L)}{I_{p}(0)}$ & $\frac{\Delta \nu_{p}(L)}{\Delta \nu_{s}(0)}$ \\
\hline 3.68 & 4.34 & 20.65 & 4.25 & 0.1174 & 0.0822 & 0.0410 & 210.0 \\
4.36 & 4.56 & 16.30 & 4.23 & 0.0944 & 0.0661 & 0.0330 & 312.0 \\
5.50 & 4.56 & 11.95 & 4.25 & 0.0500 & 0.0350 & 0.0174 & 381.0 \\
\hline $4.36^{*}$ & 4.51 & 12.97 & 4.62 & 0.0759 & 0.0531 & 0.0265 & 347.0 \\
$4.36^{* *}$ & 4.54 & 9.72 & 4.62 & 0.0256 & 0.0179 & 0.0089 & 467.0 \\
\hline
\end{tabular}

input pump bandwidth $\Delta \nu_{p}(0)$, output pump bandwidth $\Delta \nu_{p}(L)$, backward signal bandwidth $\Delta \nu_{s}(0)$, forward idler bandwidth $\Delta \nu_{i}(L)$, pump depletion or parametric downconversion efficiency $1-I_{p}(L) / / I_{p}(0)$, signal conversion $I_{s}(0) / I_{p}(0)$, idler conversion $I_{i}(L) / I_{p}(0)$, and signal coherence gain $\Delta \nu_{p}(L) / \Delta \nu_{s}(0)$. 


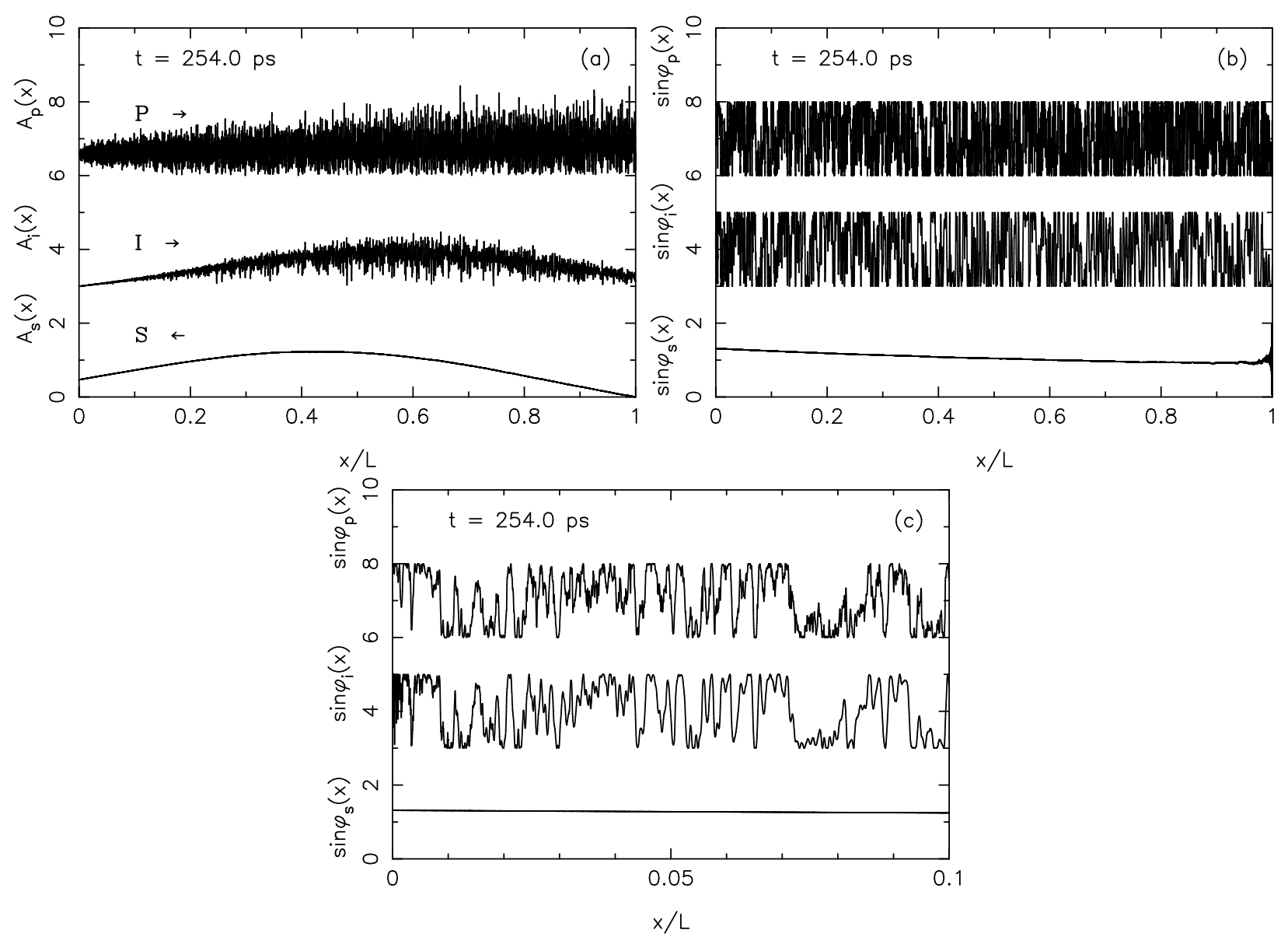

Fig. 3. (a) Amplitude distributions inside the 64 times segmented GaN BMOPO for the forward stochastic pump, the forward induced stochastic idler and the resulting coherent backward signal; (b) phase-locking inside the entire BMOPO length L, and (c) zoomed in the first L/10 length, near the maximum of the three-wave interaction (at time $t=254 \mathrm{ps}$ ). 

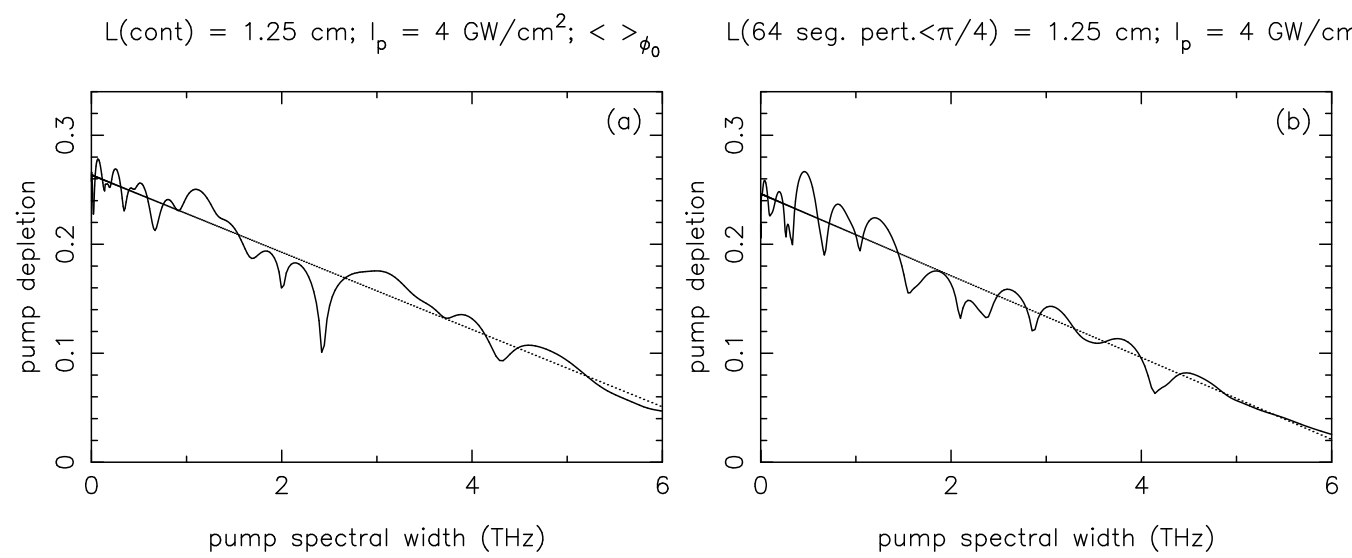

Fig. 4. Parametric downconversion efficiency [pump depletion $1-I_{p}(L) / I_{p}(0)$ ] versus input pump spectral width : (a) for the one piece perfect PPGaN BMOPO, and (b) for the 64 times segmented PPGaN BMOPO with the random phase perturbation in the junctions of amplitude $0<\delta \Phi_{m}(x) \leq \pi / 4$. The linear fitting presents a decreasing slope of -0.0375 and relative efficiency of $93 \%$ with respect to the one piece perfect PPGaN BMOPO of Fig 4(a).

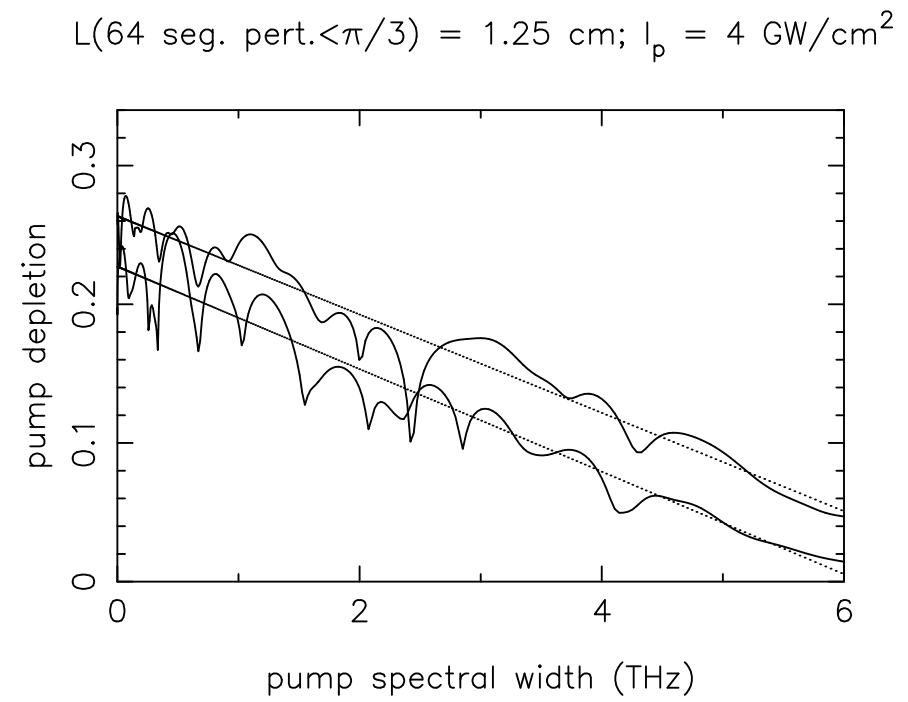

Fig. 5. Parametric downconversion efficiency [pump depletion $1-I_{p}(L) / I_{p}(0)$ ] versus input pump spectral width, for the 64 times segmented PPGaN BMOPO with the random phase perturbation in the junctions of amplitude $0<\delta \Phi_{m}(x) \leq \pi / 3$. The linear fitting presents a decreasing slope of -0.0369 and relative efficiency of $87 \%$ with respect to the one piece perfect PPGaN BMOPO (upper curve). 


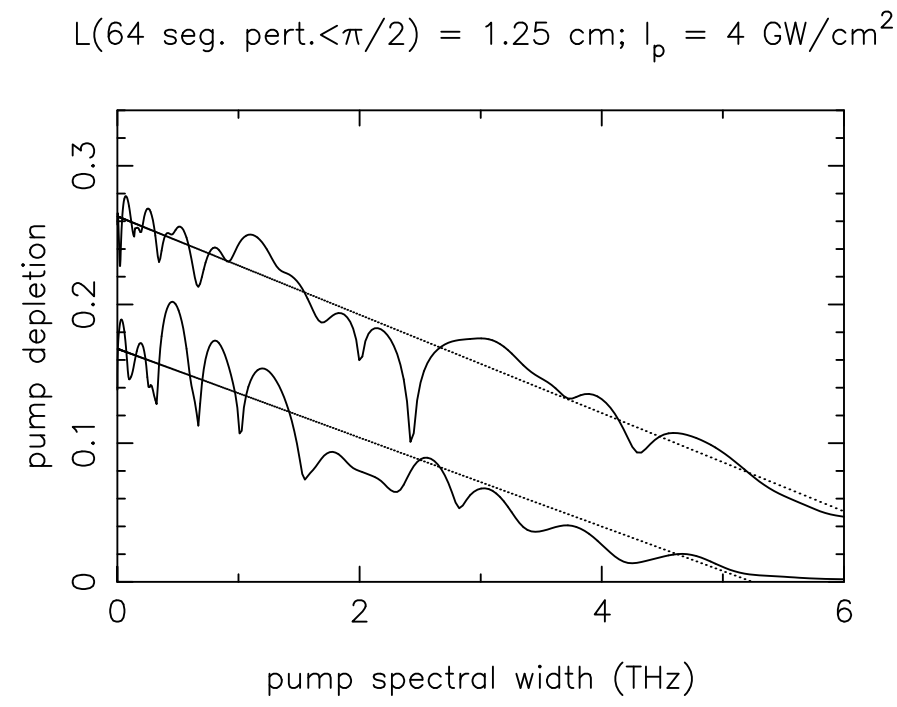

Fig. 6. Parametric downconversion efficiency [pump depletion $1-I_{p}(L) / I_{p}(0)$ ] versus input pump spectral width, for the 64 times segmented PPGaN BMOPO with the random phase perturbation in the junctions of amplitude $0<\delta \Phi_{m}(x) \leq \pi / 2$. The linear fitting presents a decreasing slope of -0.0320 and relative efficiency of $63 \%$ with respect to the one piece perfect PPGaN BMOPO (upper curve).
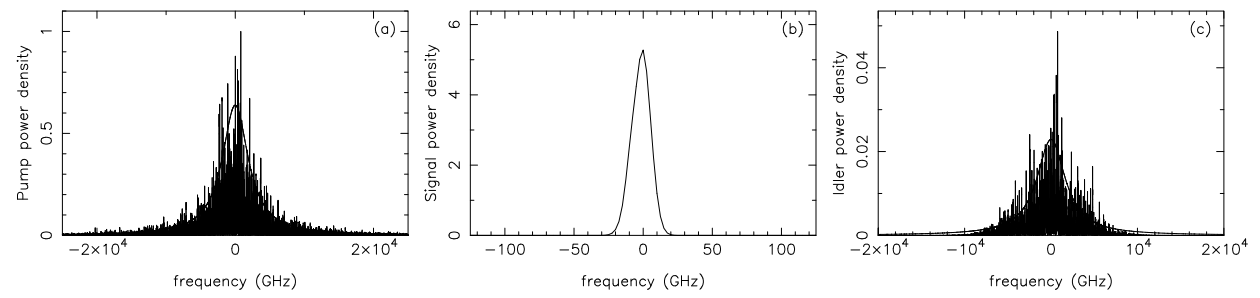

Fig. 7. Stitched BMOPO (segmented in 64 elements with maximum perturbation $\left.\delta \phi_{m}(x)=\pi / 4\right)$ for a stochastic pump input of FWHM $\Delta \nu_{p}(0)=4.36 \mathrm{THz}$, (a) Output incoherent pump spectrum fitted by a Gaussian envelope of FWHM $\Delta \nu_{p}(\mathrm{~L})=4.25 \mathrm{THz}$, (b) backward signal spectrum with $\Delta \nu_{s}(0)=16.30 \mathrm{GHz}$, and (c) forward idler spectrum with a Gaussian fitting of FWHM $\Delta \nu_{i}(\mathrm{~L})=4.23 \mathrm{THz}$. The coherence gain attains $\Delta \nu_{p} / \Delta \nu_{s}=312$ for a pump depletion or efficiency of 0.0944 (cf. Table 1). 

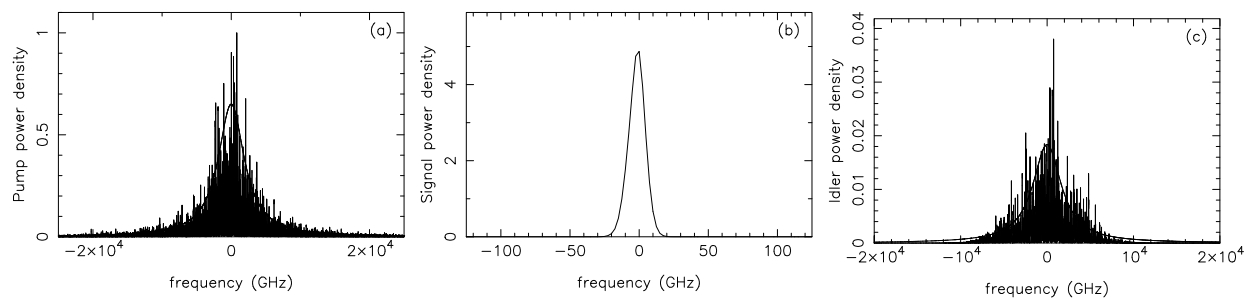

Fig. 8. Stitched BMOPO (segmented in 64 elements with maximum perturbation $\left.\delta \phi_{m}(x)=\pi / 3\right)$ for a stochastic pump input of FWHM $\Delta \nu_{p}(0)=4.36 \mathrm{THz}$ : (a) Output incoherent pump spectrum fitted by a Gaussian envelope of FWHM $\Delta \nu_{p}(\mathrm{~L})=4.51 \mathrm{THz},(\mathrm{b})$ backward signal spectrum with $\Delta \nu_{s}(0)=12.97 \mathrm{GHz}$, and (c) forward idler spectrum with a Gaussian fitting of FWHM $\Delta \nu_{i}(\mathrm{~L})=4.62 \mathrm{THz}$. The coherence gain attains $\Delta \nu_{p} / \Delta \nu_{s}=347$, for a pump depletion or efficiency of 0.0759 (cf. Table 1).
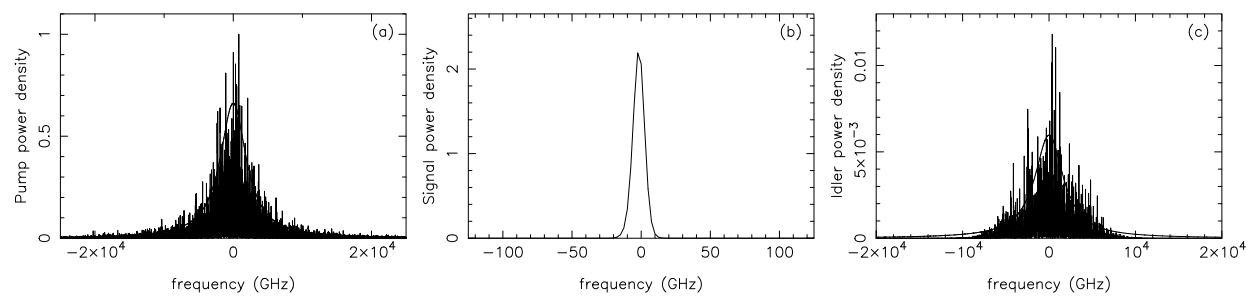

Fig. 9. Stitched BMOPO (segmented in 64 elements with maximum perturbation $\delta \phi_{m}(x)=\pi / 2$ ) for a stochastic pump input of FWHM $\Delta \nu_{p}(0)=4.36 \mathrm{THz}$ : (a) Output incoherent pump spectrum fitted by a Gaussian envelope of FWHM $\Delta \nu_{p}(\mathrm{~L})=4.54 \mathrm{THz}$, (b) backward signal spectrum with $\Delta \nu_{s}(0)=9.72 \mathrm{GHz}$, and (c) forward idler spectrum with a Gaussian fitting of FWHM $\Delta \nu_{i}(\mathrm{~L})=4.62 \mathrm{THz}$. The coherence gain attains $\Delta \nu_{p} / \Delta \nu_{s}=467$, for a pump depletion or efficiency of 0.0256 (cf. Table 1). 


\section{Summary}

We have numerically simulated the dynamics of downconversion efficiency and coherence signal enhancement in fragmented BMOPOs formed of a realistic PPGaN waveguide by using stochastic pump pulses from $0.08 \mathrm{GHz}$ to $6.0 \mathrm{THz}$ input bandwidth. We have performed the study with the original quasi-phase-matching model of Eqs.(1), which allow to consider finite phase perturbations in the junctions between the PPGaN segments in order to account for possible stitching faults. This realistic model assess the limits of the phenomenon of coherence enhancement when the phase perturbations approach half of the poling period because the parametric downconversion is replaced by sum frequency, its reverse process. For stitching errors less than the quarter of the grating period (i.e. less than $80 \mathrm{~nm}$ ), which is technically acceptable, the efficiency remains comparable to the perfect PP BMOPO, and we may obtain a coherence gain of almost three orders of magnitude.

\section{Acknowledgements}

The authors thank Pascal Baldi and Krishna Thyagarajan for stimulating discussions.

This work was granted access to the HPC and visualization resources of "Centre de Calcul Interactif" hosted by "Université Nice Sophia Antipolis". 


\section{References}

1. S. E. Harris, "Proposed backward wave oscillation in the infrared", Appl. Phys. Lett. 9, 114-116 (1966).

2. Y.I. Ding, J.B. Khurgin, "Backward optical parametric oscillators and amplifiers", IEEE J. Quantum Electron. 32, 1574-1562 (1996).

3. C. Canalias, V. Pasiskevicius, "Mirrorless optical parametric oscillator", Nature Photon. 1, 459-462 (2007).

4. G. Strömqvist, V. Pasiskevicius, C. Canalias and C. Montes, "Coherent phasemodulation transfer in counterpropagating parametric down-conversion", Phys. Rev. A 84, 023825 (2011).

5. G. Strömqvist, V. Pasiskevicius, C. Canalias, P. Aschieri, A. Picozzi, and C. Montes, "Temporal coherence in mirrorless optical parametric oscillators", J. Opt. Soc. Am. B 29, 1194-1202 (2012).

6. A. Chowdhury, H. M. Ng, M. Bhardwaj, and N. G.Weimann, "Second-harmonic generation in periodically poled GaN", Appl. Phys. Lett. 83, 1077-1079 (2003).

7. S. Pezzagna, P. Vennéguès, N. Grandjean, A. D. Wieck, and J. Massies, "Submicron periodic poling and chemical patterning of GaN", Appl. Phys. Lett. 87, 062106 (2005).

8. S. Pezzagna, J. Brault, M. Leroux, J. Massies, and M. de Micheli, "Refractive indices and elasto-optic coefficients of GaN studied by optical waveguiding", J. Appl. Phys. 103, 123112-1-7 (2008).

9. K. Gallo and G. Assanto, "All-optical diode based on second-harmonic generation in an asymmetric waveguide", J. Opt. Soc. Am. B, 16, 267-269 (1999).

10. S. H. H. Subramani, K. Karthikeyan, A. Mirunalini, R. K. Prasath, S. Boomadevi, and K. Pandiyan, "Analysis of a phase reversal quasi-phase matching device for the dual peak second harmonic response", J. Opt. 15, 055205-1-6 (2013).

11. J.A. Armstrong, N. Bloembergen, J.Ducuing, and P.S. Pershan, "Interaction between light waves in a nonlinear dielectric", Phys. Rev. A , 127, 1918-1939 (1962).

12. L. E. Myers, R. C. Eckardt, M. M. Fejer, and R. L. Byer, "Quasi-phase-matched optical parametric oscillators in bulk periodically poled LiNbO3", J. Opt. Soc. Am. B, 12, 2102-2116 (1995).

13. C. Montes, P. Aschieri, and M. de Micheli, "Backward optical parametric efficiency in quasi-phase-matched GaN waveguide presenting stitching faults", Opt. Lett. 38, No12, 2083-2085 (2013).

14. A. Picozzi and M. Hælterman, "Parametric Three-Wave Soliton Generated from Incoherent Light", Phys. Rev. Lett. 86, 2010 (2001). 
15. A. Picozzi, C. Montes, M. Hælterman, "Coherence properties of the parametric threewave interaction driven from an incoherent pump", Phys. Rev. E 66, 056605-1-14 (2002).

16. C. Montes, A. Picozzi, K. Gallo, "Ultra-coherent output from an incoherent cw-pumped singly resonant optical parametric oscillator", Opt. Commun. 237, 437-449 (2004).

17. Picozzi A and Aschieri P 2005, "Influence of dispersion on the resonant interaction between three incoherent waves", Phys. Rev. E 72, 046606-1-12

18. C. Montes, W. Grundkötter, H. Suche, and W. Sohler, "Coherent signal from incoherently cw-pumped singly resonant Ti:LiNbO3 integrated optical parametric oscillators", J. Opt. Soc. Am. B 24, 2796-2806 (2007).

19. V. Pyragaite, A. Stabinis, A. Piskarskas, and V. Smilgevicius, "Parametric amplification in the field of incoherent ligth", Phys. Rev. A 87, 063809-1-9 (2013).

20. V. Pyragaite, V. Smilgevicius,, R. Butkus, A. Stabinis, and A. Piskarskas, "Conversion of broadband incoherent pump to narrowband signal in an optical parametric amplifier", Phys. Rev. A 88, 023820-1-5 (2013).

21. A. Piskarskas, V. Pyragaite, and A. Stabinis, "Generation of coherent waves by frequency up-conversion and down-conversion of incoherent light3, Phys. Rev. A 82, 053817-1-7 (2010).

22. S. Wabnitz, A. Picozzi, A. Tonello, D. Modotto, and G. Millot, "Control of signal coherence in parametric frequency mixing with incoherent pumps: narrowband mid-infrared light generation by downconversion of broadband amplified spontaneous emission source at 1550 nm", J. Opt. Soc. Am. B, 29, 3128-3135 (2012).

23. M. Monerie, "Propagation in doubly clad single-mode fibers", IEEE J. Quantum Electron. 18, 535 (1982).

24. M. Abe, H. Sato, I. Shoji, J. Suda, M. Yoshimura, Y. Kitaoka, Y. Mori, and T. Kondo, "Accurate measurement of quadratic nonlinear-optical coefficients of gallium nitrid", J. Opt. Soc. Am. B 27, 2026-2034 (2010).

25. S. Helmfrid and G. Arvidsson, "Influence of randomly varying domain lengths and nonuniform effective index on second-harmonic generation in quasi-phase-matching waveguides", J. Opt. Soc. Am. B 8, 797-804 (1991).

26. C. Conti, E. D'Asaro, S. Stivala, A. Busacca, and G. Assanto, "Parametric self-trapping in the presence of randomized quasi phase matching", Opt. Lett. 35, No22, 3760-3762 (2010).

27. M. M. Fejer, J. A. Magel, D. H. Jundt, and R. L. Byer, "Quasi-phase-matched second harmonic generation: tuning and tolerances", IEEE J. Quantum Electron. 28, 2631-2654 (1992). 
28. C. Montes, A. Mikhailov, A. Picozzi and F. Ginovart, "Dissipative three-wave structures in stimulated backscattering. I. A subluminous solitary attractor", Phys. Rev. E 55, 1086-1091 (1997).

29. C. Montes, A. Picozzi and D. Bahloul, "Dissipative three-wave structures in stimulated backscattering. II. Superluminous and subluminous solitons", Phys. Rev. E 55, 10921106 (1997).

30. C. Montes, P. Aschieri, and A. Picozzi, "Model for Coherence Transfer in a Backward Optical Parametric Oscillator", SPIE Proc. 8011, 801136-1-10 (2011).

31. F.Kh. Abdullaev, S.A. Darmanyan, A. Kobyakov, E. Schmidt, and F. Lederer, "Evolution of spatial solitons in quadratic media with fluctuating nonlinearity", Optics Commun. 168, 213-218 (1999).

32. X.C. Wang, G.C. Lim, F.L. Ng, W. Liu, and S.J. Chua, "Femtosecond pulsed laserinduced periodic surface structures on GaN/sapphire", Applied Surface Science 252, 1492-1497 (2005).

33. K. Ozono, M. Obara, A. Usui, and H. Sunakawa, "High-speed ablation etching of GaN semiconductor using femtosecond laser", Optics Commun. 189, 103-106 (2001). 